\title{
Editorial: Robust Monitoring, Diagnostic Methods and Tools for Engineered Systems
}

\author{
Manolis N. Chatzis ${ }^{1 *}$, Eleni N. Chatzi ${ }^{2}$ and Costas Papadimitriou ${ }^{3}$ \\ ${ }^{1}$ Department of Engineering Science, Mathematical, Physical and Life Sciences Division, University of Oxford, Oxford, \\ United Kingdom, ${ }^{2}$ Department of Civil Environmental and Geomatic Engineering, ETH Zürich, Zurich, Switzerland, \\ ${ }^{3}$ Department of Mechanical Engineering, University of Thessaly, Volos, Greece
}

Keywords: monitoring, damage identification, data-driven models, diagnostic tools, uncertainty quantification, structural health monitoring

\section{Editorial on the Research Topic}

\section{Robust Monitoring, Diagnostic Methods and Tools for Engineered Systems}

Complex engineered systems manifest across all engineering fields. Such systems are further characterized by uncertainties linked to assumptions and limited information on material constitutive laws, description of loads, the influence of operational and environmental factors, energy dissipation mechanisms, motion constraints, or large displacements of system components. The propagation of these uncertainties adversely affects simulation accuracy and, consequently,

OPEN ACCESS

Edited by:

Nizar Bel Hadj Ali,

École Nationale D'Ingénieurs de

Gabès, Tunisia

Reviewed by:

Eliz-Mari Lourens,

Delft University of

Technology, Netherlands

*Correspondence:

Manolis N. Chatzis

manolis.chatzis@eng.ox.ac.uk

Specialty section:

This article was submitted to Structural Sensing

a section of the journal

Frontiers in Built Environment

Received: 29 May 2020

Accepted: 08 July 2020

Published: 25 August 2020

Citation:

Chatzis MN, Chatzi EN and Papadimitriou C (2020) Editorial: Robust Monitoring, Diagnostic Methods and Tools for Engineered

Systems. Front. Built Environ. 6:125. doi: 10.3389/fbuil.2020.00125 the design, operation, and maintenance decisions for meeting desirable system performance and safety requirements.

Structural Health Monitoring exploits measurements from operating or tested systems for the development of robust diagnostic tools and procedures, which aim to improve condition assessment of complicated engineering systems under uncertainty. Researchers are pushing the boundaries of such uncertainty quantification tools and diagnostic and prognostic methods to improve the accuracy of the predictions, or for achieving robust results under sensorial information that is less accurate but better tailored to functionality requirements. The works in this special issue, deal with the previous directions.

In Mugabo et al. and Mugabo et al. an experimental campaign on a three-story timber building, the "Albina Yard," is performed using a set of accelerometers, with the dataset further made available to the scientific community. The authors further demonstrate how Operational Modal Analysis methods succeed in identifying the modal properties of this hybrid timber building under ambient excitation. The findings were compared to a finite element representation of the building and led to the interesting conclusion of how secondary-elements, such as an exterior wall, and non-structural elements could bear a significant effect in the modal properties, and therefore the dynamics, of such buildings. Such a fusion with a system model is often critical to the assessment. However, in practice, engineers need to resort to model assumptions and simplifications, which as discussed in Song et al. can result in bias. Song et al. account for this bias by identifying not only the structural parameters of the assumed model but also of the stochastic properties of the modeling error through a hierarchical Bayesian framework. This allows for removing the effects of the bias and obtaining more reliable estimates of the modal properties of the simplified model. The method is demonstrated by identifying the properties of a shear-type building using data from a building with rocking foundation.

The drive for energy-efficient sensors for continuous monitoring of field applications has brought forth challenges related to the acquired data. In Horner et al. the authors discuss the effect of missing observations in estimating the parameters of regression models and 
suggest a novel methodology to efficiently do so. Experimental data from a two-bay steel frame and simulated data are used illustrating that the method can operate robustly despite a significant amount of missing data. In Gkoktsi et al. the challenges presented by measurements obtained at a lower sampling frequency, for example resulting from compression at the wireless nodes of field sensors, are addressed. The authors suggest two methods to cope with sub-Nyquist and non-uniformly sampled time histories and demonstrate the reconstruction of the original signals in the frequency and time domain. Experimental data from a monitored highway bridge and an on-shore Wind Turbine are used to demonstrate the ability of the methods to robustly reconstruct the signals for output-only identification.

A challenge in the monitoring of field structures often lies in the influence of the environmental and operational conditions, which can result in challenging the commonly used assumption of time-invariance. In Avendaño-Valencia et al. the authors address this challenge for the effect of variable wind speeds on Wind Turbines. Using Gaussian processes, the coefficients of auto-regressive models representing the structure are updated for variable wind speeds. The authors demonstrate the capacity of the method in terms of estimating the fatigue life of a wind turbine. Similarly, in Gislason et al. the authors rely on the use of autoregressive time series models for identifying damage in structural buildings. This is achieved via coupling of ARMAX models with a sensor clustering concept, for use with ambient vibration sensors, such as accelerometers. The authors demonstrate that the changes in the properties of such timeseries models would be able to detect damage in structures as demonstrated in simulated examples of multi-story buildings.

Strain measurements are revealed as a valuable tool for condition assessment, and estimation of reserve capacity. In this context Kliewer and Glisic employ a series of long-gauge Fiber Bragg Grating sensors for damage detection in beam-types of structures, by means of a so-called normalized curvature ratio. The method is demonstrated to robustly detect damage along beam-type structures, or changes in the support conditions in analytical examples, an experimental test and when used on field data from a highway bridge. In separate work exploiting strains, the fatigue life of the Venoge Bridge is the topic of investigation of Pai et al. relying on deployment of strain sensors placed on the bridge since 1995. The authors investigate different methods for updating a Finite Element model of the Bridge. A modified Bayesian updating scheme is proposed, which explicitly includes model bias, and a model falsification framework (EDMF) are implemented and cross-assessed for updating the model parameters, which in turn allows estimating the remaining fatigue life of the structure. The authors further suggest that EDMF offers the additional advantage of compatibility with engineering practice.

A mechanical engineering application is the focus in Matthaiou et al. where the authors target condition monitoring for gas turbines. The method presented by the authors is data-driven, using a machine-learning approach based on novelty detection, focusing on the utilization of training data that correspond to mainly healthy cases. The framework is demonstrated on experimental vibration data from engines operating on different types of fuel, proving the diagnostic capability of the method. Remaining in the context of diagnostics, a modification of the Unscented Kalman Filter for the case of non-smooth systems is presented in Chatzis and Chatzi, termed the Discontinuous UKF. Non-smooth systems arise from the mathematical representations of phenomena related to damage such as sliding, impacts or plasticity. The authors demonstrate in numerical examples how the Discontinuous modification allows for detecting the properties of such systems and achieve damage detection in a robust and online manner. In Abdessalem et al. the authors present a novel combination of two Bayesian tools, Gaussian Processes (GPs), and the use of the Approximate Bayesian Computation (ABC) algorithm for kernel selection and parameter estimation in machine learning applications. The method is demonstrated on simulated and actual datasets.

The previously mentioned papers present a series of tools that deliver information on the condition of an asset and, in some cases, further allow estimating its remaining lifespan. A common issue is how such information can be utilized by a managing authority for the process of decision making. This is discussed in Aktan et al., where the authors present an overview of how sensorial information can be exploited by managing authorities and a roadmap for facilitating such a transition in asset management through appropriate training. In rendering further linkage to the practice of construction, Singh and Sadhu deliver a dynamic Building Information Modeling (BIM) webbased framework, which incorporates online visualization of data, real-time system identification, and decision-making. A steel bridge located in London, Ontario is utilized as a case study, where both BIM and SHM are integrated in a unified fashion.

Despite the obvious hurdles posed by uncertainties in the monitoring and diagnostics of engineered systems, the works featured in this Special Issue clearly demonstrate that adoption of a data-driven attitude toward structural assessment is not only the way forward, but also mature enough to be put into practice.

\section{AUTHOR CONTRIBUTIONS}

MC prepared the original draft with contributions from EC. EC and $\mathrm{CP}$ revised and $\mathrm{MC}$ submitted the final version. All authors contributed to the article and approved the submitted version.

\section{ACKNOWLEDGMENTS}

The editors would like to thank to all contributors to this special issue as well as the reviewers and editorial team of Frontiers.

Conflict of Interest: The authors declare that the research was conducted in the absence of any commercial or financial relationships that could be construed as a potential conflict of interest.

Copyright () 2020 Chatzis, Chatzi and Papadimitriou. This is an open-access article distributed under the terms of the Creative Commons Attribution License (CC BY). The use, distribution or reproduction in other forums is permitted, provided the original author(s) and the copyright owner(s) are credited and that the original publication in this journal is cited, in accordance with accepted academic practice. No use, distribution or reproduction is permitted which does not comply with these terms. 